\title{
A feasible method to improve adherence of Hawley retainer in adolescent orthodontic patients: a randomized controlled trial
}

This article was published in the following Dove Press journal:

Patient Preference and Adherence

28 October 2015

Number of times this article has been viewed

\author{
Feiou Lin' \\ Hao Sun' \\ Zhenyu $\mathrm{Ni}^{\prime}$ \\ Minling Zheng' \\ Linjie Yao² \\ 'Orthodontic Department, \\ ${ }^{2}$ Pedodontic Department, School \\ of Stomatology, Wenzhou Medical \\ University, Wenzhou, Zhejiang, \\ People's Republic of China
}

Background: Retention is an important component of orthodontic treatment; however, poor compliance with retainer use is often encountered, especially in adolescents. The purpose of this study was to prove the hypothesis that verbal instructions combined with images showing the severe consequences of poor compliance can increase retainer use.

Methods: This study was a randomized controlled trial. The sample was recruited from Wenzhou, People's Republic of China, between February 2013 and May 2014, and 326 participants were randomized into three groups. Patients and parents in Group A $(n=106)$ were given routine retainer wear instructions only; in Group B ( $n=111)$, images illustrating the severe consequences of poor compliance with Hawley retainer use were shown to patients, combined with routine instructions; and in Group C ( $\mathrm{n}=109)$, images illustrating the severe consequences of poor compliance with Hawley retainer use were shown to patients and parents, combined with routine instructions. Three months after debonding, questionnaires were used to investigate daily wear time and the reasons for poor compliance. Differences in means between the groups were tested by one-way analysis of variance.

Results: The mean daily wear time in Group C (15.09 \pm 4.13 hours) was significantly greater than

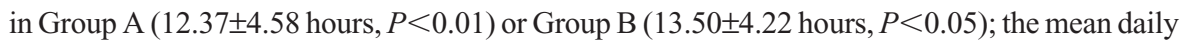
wear time in Group B was greater than in Group A, but was not significant $(P=0.67)$. Reasons for nonusage were forgetting to wear the retainer (51\%) and finding the retainer bothersome to frequently insert and remove (42\%).

Conclusion: Verbal instructions combined with images showing the severe consequences of poor compliance can increase retainer use. Parents play an important role in compliance with retainer use in adolescent patients.

Keywords: orthodontics, Hawley retainers, adherence

\section{Introduction}

It is well know that orthodontic treatment can improve an individual's appearance, oral function, psychosocial well-being, and quality of life. ${ }^{1-3}$ After orthodontic treatment, retainers are used to hold teeth in the correct position; however, several long-term studies have shown that after standardized orthodontic treatment, relapse occurs in approximately $70 \%$ of all cases. ${ }^{4-6}$ This is mainly related to the influence of the periodontal and gingival tissues, the unstable position of teeth, and continued skeletal growth. ${ }^{5}$ Retention of orthodontic repositioning is a critical component of successful long-term treatment. To solve these problems, retainers are used to prevent relapse until gingival and periodontal reorganization and skeletal growth are completed. Various types of retainers are applied, including fixed retainers, removable Hawley retainers, and vacuum-formed retainers. ${ }^{7-9}$ Most orthodontists prescribe 6 months of full-time wear of a removable retainer followed 
by part-time wear for life. ${ }^{10}$ It is well known that patient compliance with wearing Hawley retainers is particularly important for retention, because noncompliance can destroy the best treatment outcomes. In cases in which the long-term treatment outcome is unsatisfactory, it is difficult to evaluate to what extent noncompliance contributed, or whether the failure was caused by biological factors. Many researchers have focused on identifying factors correlated with compliance and cooperation. Nanda and Kierl ${ }^{11}$ attempted to predict patients' compliance with retainer use by measuring attitudes toward treatment, social desirability, need for approval, and need for achievement. None of these variables adequately predicted the cooperation of a patient with orthodontic treatment. Mehra et $\mathrm{al}^{12}$ found that patient-related items, such as desire for treatment and relationship with parents, ranked as important factors motivating patients to comply. Verbal praise and communication are rated as important methods for improving compliance. Egolf et $\mathrm{al}^{13}$ found that the pain and inconvenience associated with intraoral elastics or headgear were inversely correlated with compliance. These findings may also apply to retainer wear.

Many studies have investigated suitable methods for measuring a patient's removable appliance wear time, ${ }^{14,15}$ so quantifying patient compliance over the whole therapy period can routinely be performed in an orthodontic office. ${ }^{16-18}$ However, few studies have researched the factors that predict compliance with retainer use or interventions that improve compliance with removable appliances or retainers. ${ }^{13}$ Patient compliance was found to be influenced by psychological and sociodemographic traits. ${ }^{19}$ Changes in patients' knowledge about the severe consequences of poor compliance could alarm them and increase their retainer use. The primary aim of this clinical trial was to test the effectiveness of verbal instructions combined with images showing the severe consequences of poor compliance.

\section{Methods}

Ethical approval for the study was granted by the Health Research Ethics Board at Wenzhou Medical University (WYKQ2014002). Each patient and parent was given oral and written information and had to provide written consent before being included in the trial. The research was designed as a prospective, randomized controlled three-arm parallel trial (Figure 1). The sample size for each group was calculated as $n=97$ (ie, a total of 288 for three groups), based on type II error at 0.10 ( $90 \%$ power), with a 5\% significance level to detect a difference of 2 hours of daily wear time ( $\mathrm{SD}=4.2$ hours according to previous estimates). To account for possible dropouts and loss to follow-up during the study, we recruited 120 patients in each group. The final

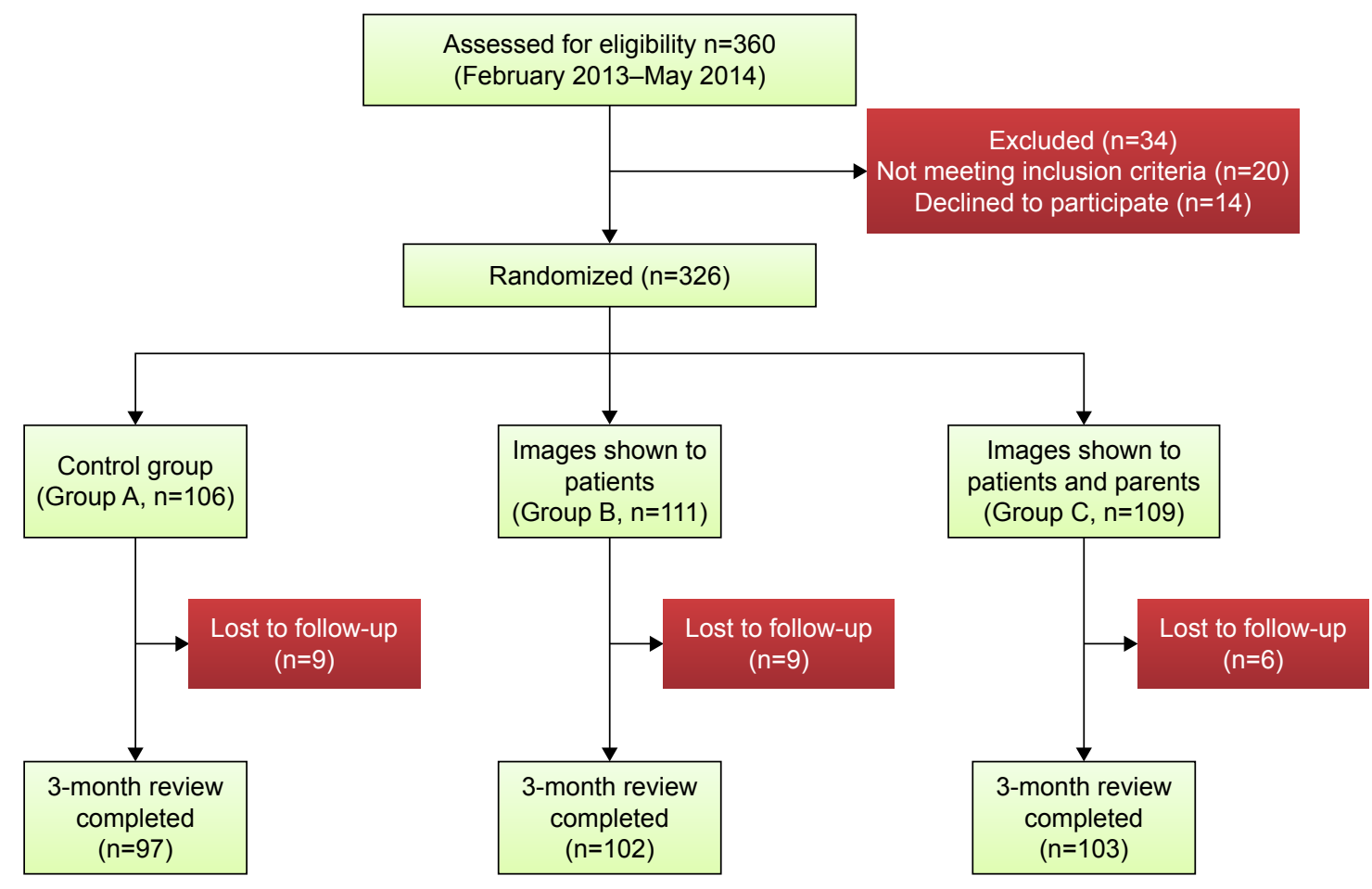

Figure I Flow chart of the trial.

Notes: Group A: control group; Group B: patients shown images illustrating the severe consequences of poor compliance with Hawley retainer use; Group C: patients and parents shown images illustrating the severe consequences of poor compliance with Hawley retainer use. 
number of patients completing the study was 302 patients from Wenzhou, People's Republic of China (age range, 11-18 years), wearing Hawley retainers.

The subjects were recruited for this pilot study between February 2013 and May 2014, based on specific inclusion and exclusion criteria. Patients fulfilling the following criteria were included: age range 11-18 years, fixed appliance involving both arches, under treatment before recruitment, and willing to wear a Hawley retainer. Patients with the following conditions were excluded: single-arch or sectional fixed appliance treatment, learning difficulties, cleft lip or palate, craniofacial syndrome, or requirement for tooth replacement on the retainer as a temporary measure.

A simple randomization with no blocking was used in this research. Eligible patients were randomly assigned to three groups based on computer-generated random sequencing. To avoid subjective bias, we applied a double-blind method. The patients did not know their allocation, and the investigator was blinded to the allocation. Three trained researchers introduced the visual method to the participants and their parents according to the random sequencing. In view of the ethical issues and patients' equal rights for oral health education, all patients and parents received routine instructions about retainer wear. The parents/guardians who were living full-time with the patients were asked to receive instructions of retainer wear at the time of recruitment.

The patients were randomly assigned to one of three intervention groups (Figure 1). Group A (control group) was provided with routine retainer wear instructions only; in Group B, images illustrating the severe consequences of poor compliance with Hawley retainer use were shown to patients (Figure 2); and in Group C, images illustrating the severe consequences of poor compliance with Hawley retainer use were shown to patients and parents.
According to orthodontic researches and clinic, crowding and space relapse were the most common relapses, and most patients were concerned about it. Thus, we chose classic relapse images (Figure 2) from our clinic to show to the patients.

The routine instructions about retainer wear included chairside verbal instructions, a written explanation of recommended wear time, and verbal information for the patient and parents about the results of poor compliance with Hawley retainer use. When the investigator showed the images illustrating the severe consequences of poor compliance with Hawley retainer, he told the treatment history of these two patients and pointed out the relapse in the picture; meantime, he warned the patients about the severe consequences of poor compliance according to their own conditions. The retention protocol required the patients to wear Hawley retainers full-time after debonding and to revisit after 3 months. Full-time compliance meant that patients should wear the retainer at all times except when eating or toothbrushing.

A questionnaire (Figure 3), with a cover letter explaining the purpose of the study, was used to identify how long retainers were worn in the 2 weeks before first revisit and any reasons for not wearing the retainers as instructed. The list of reasons was compiled from interviews with 50 patients who previously wore retainers, and was approved by three professors of orthodontics. After the patient completed the questionnaire, we checked the accuracy of the wear time response by communicating with the parents living with the patient. SPSS software (version 17, SPSS, Chicago, IL, USA) was used to calculate frequencies and percentages and to run one-way analysis of variance (ANOVA) to detect differences in compliance among the three groups. The significance level $(\alpha)$ for ANOVA was set at $P<0.05$.
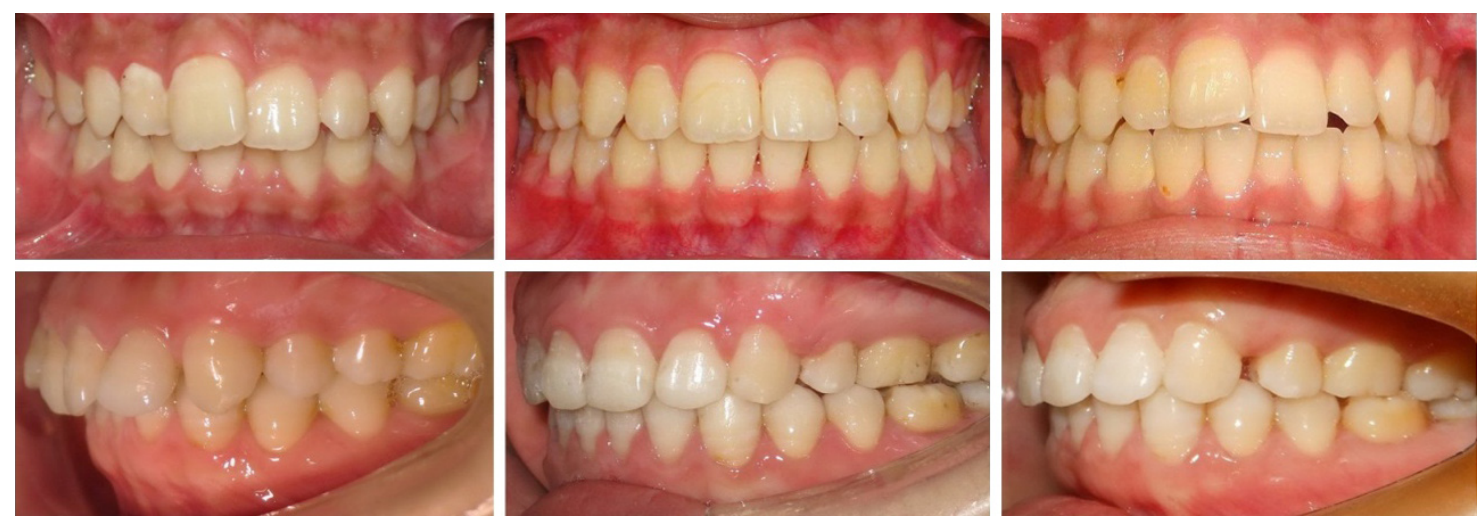

Figure 2 Images shown to patients and parents.

Notes: Images illustrating the consequences of poor compliance with Hawley retainer use, including crowding and space relapse, were shown to patients in Group B and to patients and their parents in Group C. The left two images show the pretreatment condition, images in the middle show the result of a treatment, and right images show relapse. 
The following survey is part of a research conducted by the Department of

Orthodontics at the Wenzhou Medical University. Your comments will provide

valuable information for the research. Please take several minutes and fill out the

questions below. No one will know who completed this questionnaire, by completing

and returning this survey, you are signifying that you understand this study and

consent to participate in it.

1. Gender: ___ Age:

2. Please recall the wear-time in the last 2 weeks and make a mark on the line below to indicate how long do you wear your retainer daily?

$\begin{array}{lrrrr} & 1 & 1 & 1 & \\ \text { Never } & 6 \mathrm{~h} & 12 \mathrm{~h} & 18 \mathrm{~h} & 24 \mathrm{~h}\end{array}$

3. If you are wearing retainers less than 18 hours, which of the following reasons do you agree with? (check all that apply)
$\square$ It is uncomfortable
$\square$ I lost it
$\square$ I forget to wear it
$\square$ It affects my appearance
$\square$ I do not think it is important
$\square$ I have a lisp wearing retainers
$\square$ It affects my pronunciation
$\square$ Other people affect my compliance
$\square$ It is bothersome frequently inserting and removing the retainer
$\square$ Other

Figure 3 Questionnaire about retainer use.

Note: This questionnaire about retainer use and reasons for noncompliance was issued to patients 3 months after active treatment.

Abbreviation: $\mathrm{h}$, hours.

\section{Results}

The demographic characteristics of the participants are shown in Table 1. Figure 1 shows the CONSORT diagram of the clinical trial. Three hundred and sixty patients were recruited and assessed for eligibility. Twenty patients did not meet the inclusion criteria, and 14 patients declined to join in the trial. A total of 326 patients were recruited for the randomized controlled trial (Group A, 106; Group B, 111; Group C, 109); after 3 months of retention, 24 patients (Group A, 9; Group B, 9; Group C, 6) were lost to follow-up because of moving to other places or being unwilling to return for review. Table 2 shows the mean daily wear time of the three groups. The mean daily wear time in Group C (15.09 \pm 4.13 hours) was significantly greater than that in Group A $(12.37 \pm 4.58$ hours, $P<0.01)$ and Group B $(13.50 \pm 4.22$ hours, $P<0.05)$. The mean daily wear time in

Table I Demographic characteristic of the participants

\begin{tabular}{llll}
\hline Demographic & Group A & Group B & Group C \\
\hline Age (years) & & & \\
II-14 & 55 & 62 & 59 \\
I5-18 & 42 & 40 & 44 \\
Sex & & & \\
Male & 39 & 38 & 45 \\
Female & 58 & 64 & 58 \\
Malocclusion & & & \\
Class I & 48 & 49 & 5 I \\
Class II & 42 & 42 & 44 \\
Class III & 7 & 1 I & 8 \\
Total & 97 & 102 & 103 \\
\hline
\end{tabular}

Group B was greater than in Group A, but was not significant $(P>0.67)$.

A total of 244 patients wore retainers for less than 18 hours per day for reasons listed in Table 3. The top three reasons for poor compliance were: "I forget to wear it" (51.2\%); "It is bothersome frequently inserting and removing the retainer" (41.8\%), and "I do not think it is important" (32\%). Other reasons for poor compliance related to the retainer being uncomfortable and affecting appearance and pronunciation.

\section{Discussion}

Approaching positive targets and avoiding negative ones is a central requirement in motivation, and avoidance of harmful things reflects a fundamentally evolved motive that influences the way people perceive and respond to their social world. ${ }^{20}$ The motivation to seek orthodontic treatment is complicated; however, it has been proven that the desire for better dental esthetics is one of the most important motivations. ${ }^{21-23}$ When motivation is lost, there is a danger that a patient's compliance will suffer, and this could lead to

Table 2 The mean daily wearing time in the different groups

\begin{tabular}{lll}
\hline Group & $\mathbf{n}$ & Daily wearing time $(=\overline{\mathbf{X}} \pm \mathbf{S D}, \mathbf{h})$ \\
\hline A & 97 & $12.37 \pm 4.58^{*}$ \\
B & 102 & $13.50 \pm 4.22^{* *}$ \\
C & 103 & $15.09 \pm 4.13$ \\
\hline
\end{tabular}

Notes: Group A or Group B compared with Group C, *distinguishing significant at $P<0.0$ I; **distinguishing significant at $P<0.05$. No significant variations of retainer wearing time were found between Group A and Group B ( $P>0.05)$.

Abbreviations: SD, standard deviation; h, hours. 
Table 3 The reasons of poor adherence of adolescent patients and demographics

\begin{tabular}{ll}
\hline Reasons & Total (n, \%) \\
\hline It is uncomfortable & $69(28.3)$ \\
I forget to wear it & $125(51.2)$ \\
I do not think it is important & $78(32.0)$ \\
I have a lisp wearing retainers & $46(18.9)$ \\
Other people affect my adherence & $44(18)$ \\
I lost it & $29(11.9)$ \\
It affects my appearance & $26(10.7)$ \\
It is bothersome taking off and & $102(41.8)$ \\
putting on retainers frequently & \\
It affects my pronunciation & $42(17.2)$ \\
Others & $24(9.8)$ \\
\hline
\end{tabular}

premature termination of treatment or a compromised result. External influences play an important role in motivating teenagers to undergo treatment, especially the attitudes and perceptions of family members, which provide the foundation on which a child builds his or her self-perception of dentalfacial appearance. ${ }^{22,24,25}$ Based on previous research, our study was designed to test the hypothesis that negative images can increase awareness of the results of poor retention and may improve the patient's compliance, and that cooperation from the patients' parents is also an important factor.

The results of this study suggest that patient compliance can be improved by showing severe relapse pictures to the adolescent patients, but the improvement is not significant. These findings differ slightly from the research of Peng et al, ${ }^{26}$ who educated patients about the severe consequences of biofilm accumulation using visual aids and observed significant improvements in oral hygiene. It was hypothesized that adolescents' immature mentality and lack of knowledge about orthodontic treatment (especially, the importance of retention) were the main reasons for poor compliance. ${ }^{27,28}$ In our study, the highest level of compliance was found in Group C, in which the adolescent patients' parents were shown images of severe relapse. This finding, demonstrating that parents play an important role in the cooperation of adolescent patients, is consistent with the results of previous research. ${ }^{27,28}$ The median wear time was 12.4 hours per day in Group A, which is higher than in previous studies..$^{29,30}$ This could be because of differences in the study population and timing methods. Previous research has suggested that subjective factors could result in overestimation of compliance, and patients' feedback about retainer wear time tends to be subjective; ${ }^{31}$ therefore, future studies should incorporate the use of an objective instrument to measure retainer compliance.

In this research, we identified barriers to compliance with removable retainer use during orthodontic treatment. The major reasons for poor cooperation in adolescent patients included: "I forget to wear it"; "It is bothersome frequently inserting and removing the retainer"; "I do not think it is important"; and "It is uncomfortable". More than half of the subjects $(51 \%)$ regularly forgot to wear their retainer. Schott and Ludwig ${ }^{32}$ found that the majority of young patients showed discontinuous wear behavior, with a typical pattern of not wearing the device on some days and then trying to compensate for this by wearing the device more on other days. Therefore, it is important for orthodontists to do whatever they can to encourage patients to wear their retainers. Eppright et $\mathrm{al}^{33}$ found that a text message reminder system was effective for improving oral hygiene compliance in orthodontic patients, and these findings might apply equally well to retainer wear. Once the parents recognized the serious consequences of poor retention, they tended to supervise the patient and remind them to wear the retainer, which could explain why compliance was best in Group C. We should improve patient education about orthodontics to increase knowledge of the importance of retention. In some instances, we may choose a fixed retainer for certain patients to avoid the undesirable results of noncompliance arising from inconvenience or discomfort. It is notable that poor esthetics and effects on pronunciation were not found to be major reasons for poor compliance.

In general, the ability to predict treatment compliance and retainer wear behavior would be valuable in orthodontic treatment, although it is a very complex issue. ${ }^{11,12}$ Parental influence, the age of the patient, retainer design, the sex of the patient, the orthodontist's prescription, and fear or disregard of relapse are all factors that may influence patient behavior. Our research provides a feasible and effective method to improve patient compliance with retainer use, and this positive effect is consistent with studies in other areas. ${ }^{19,26}$ Most adolescent patients and parents are not profoundly aware of the severe consequences of poor retention. Verbal instruction is an auditory stimulus which works particularly well when combined with visual stimuli. ${ }^{34}$ This is why relapse images can reinforce perceptions about the results of poor retention and may lead to improved patient compliance. The two main limitations of this study were that it used a subjective method to measure compliance, which could have resulted in an overestimation of compliance, ${ }^{31}$ and that all the patients were from Zhejiang Province, People's Republic of China, and they had a relatively low level of knowledge about orthodontic retention compared with patients from developed countries.

\section{Conclusion}

1. Verbal instructions combined with images showing the severe consequences of poor compliance can increase retainer use. 
2. Parents play an important role in the adherence of retainer in adolescent patients.

3. The major reasons for poor compliance with retainer use were that patients forget to wear it and find it bothersome to insert and remove.

\section{Disclosure}

The authors report no conflicts of interest in this work.

\section{References}

1. Perillo L, Esposito M, Caprioglio A, Attanasio S, Santini AC, Carotenuto M. Orthodontic treatment need for adolescents in the Campania region: the malocclusion impact on self-concept. Patient Prefer Adherence. 2014;8:353-359.

2. Perillo L, Esposito M, Contiello M, Lucchese A, Santini AC, Carotenuto M. Occlusal traits in developmental dyslexia: a preliminary study. Neuropsychiatr Dis Treat. 2013;9:1231-1237.

3. Feu D, de Oliveira BH, de Oliveira Almeida MA, Kiyak HA, Miguel JA. Oral health-related quality of life and orthodontic treatment seeking. Am J Orthod Dentofacial Orthop. 2010;138(2):152-159.

4. Sadowsky C, Sakois EI. Long term assessment of orthodontic relapse. Am J Orthod. 1982;82(6):456-463.

5. Melrose C, Millett DT. Toward a perspective on orthodontic retention? Am J Orthod Dentofacial Orthop. 1998;113(5):507-514.

6. Sadowsky C, Schneider BJ, BeGole EA, Tahir E. Long-term stability after orthodontic treatment: nonextraction with prolonged retention. Am J Orthod Dentofacial Orthop. 1994;106(3):243-249.

7. Jäderberg S, Feldmann I, Engström C. Removable thermoplastic appliances as orthodontic retainers - a prospective study of different wear regimens. Eur J Orthod. 2012;34(4):475-479.

8. Singh P, Grammati S, Kirschen R. Orthodontic retention patterns in the United Kingdom. J Orthod. 2009;36(2):115-121.

9. Keim RG, Gottlieb EL, Nelson AH, Vogels DS. 2008 JCO study of orthodontic diagnosis and treatment procedures, part 1: results and trends. J Clin Orthod. 2008;42(11):625-640.

10. Valiathan M, Hughes E. Results of a survey-based study to identify the most common retention practices in the United States. Am J Orthod Dentofacial Orthop. 2010;137(2):170-177.

11. Nanda RS, Kierl MJ. Prediction of cooperation in orthodontic treatment. Am J Orthod Dentofacial Orthop. 1992;102(1):15-21.

12. Mehra T, Nanda RS, Sinha PK. Orthodontists' assessment and management of patient compliance. Angle Orthod. 1998;68(2):115-122.

13. Egolf RJ, BeGole EA, Upshaw HS. Factors associated with orthodontic patient compliance with intraoral elastic and headgear wear. Am J Orthod Dentofacial Orthop. 1990;97(4):336-348.

14. Cole WA. Accuracy of patient reporting as an indication of headgear compliance. Am J Orthod Dentofacial Orthop. 2002;121(4):419-423.

15. Kyriacou PA, Jones DP. Compliance monitor for use with removable orthodontic headgear appliances. Med Biol Eng Comput. 1997; 35(1):57-60.

16. Schott TC, Göz G. Applicative characteristics of new microelectronic sensors Smart Retainer ${ }^{\circledR}$ and TheraMon ${ }^{\circledR}$ for measuring wear time. J Orofac Orthop. 2010;71(5):339-347.

Patient Preference and Adherence

\section{Publish your work in this journal}

Patient Preference and Adherence is an international, peer-reviewed, open access journal that focuses on the growing importance of patient preference and adherence throughout the therapeutic continuum. Patient satisfaction, acceptability, quality of life, compliance, persistence and their role in developing new therapeutic modalities and compounds to optimize
17. Schott TC, Göz G. Wearing times of orthodontic devices as measured by the TheraMon ${ }^{\circledR}$ microsensor. J Orofac Orthop. 2011;72(2):103-110.

18. Schott TC, Ludwig B. Quantification of wear-time adherence of removable appliances in young orthodontic patients in relation to their BMI: a preliminary study. Patient Prefer Adherence. 2014;8:1587-1595.

19. Taylor GH, Wilson SL, Sharp J. Medical, psychological, and sociodemographic factors associated with adherence to cardiac rehabilitation programs: a systematic review. J Cardiovasc Nurs. 2011;26(3):202-209.

20. Lewin K. A Dynamic Theory of Personality. New York, NY: McGrawHill Press; 1935.

21. Daniels AS, Seacat JD, Inglehart MR. Orthodontic treatment motivation and cooperation: a cross-sectional analysis of adolescent patients' and parents' responses. Am J Orthod Dentofacial Orthop. 2009;136(6):780-787.

22. Bos A, Hoogstraten J, Prahl-Andersen B. Towards a comprehensive model for the study of compliance in orthodontics. Eur J Orthod. 2005; 27(3):296-301

23. Prabakaran R, Seymour S, Moles DR, Cunningham SJ. Motivation for orthodontic treatment investigated with Q-methodology: patients' and parents' perspectives. Am J Orthod Dentofacial Orthop. 2012;142(2):213-220.

24. Gosney MB. An investigation into some of the factors influencing the desire for orthodontic treatment. Br J Orthod. 1986;13(2):87-94.

25. Trulsson U, Strandmark M, Mohlin B, Berggren U. A qualitative study of teenagers' decisions to undergo orthodontic treatment with fixed appliance. J Orthod. 2002;29(3):197-204.

26. Peng $\mathrm{Y}, \mathrm{Qu} \mathrm{W}, \mathrm{Wu} \mathrm{W}$, et al. Effect of visual method vs plaque disclosure in enhancing oral hygiene in adolescents and young adults: a singleblind randomized controlled trial. Am J Orthod Dentofacial Orthop. 2014;145(3):280-286.

27. Bos A, Hoogstraten J, Prahl-Andersen B. The theory of reasoned action and patient compliance during orthodontic treatment. Community Dent Oral Epidemiol. 2005;33(6):419-426.

28. Albino JE, Lawrence SD, Lopes CE, Nash LB, Tedesco LA. Cooperation of adolescents in orthodontic treatment. J Behav Med. 1991; 14(1):53-70.

29. Schott TC, Schlipf C, Glasl B, Schwarzer CL, Weber J, Ludwig B. Quantification of patient compliance with Hawley retainers and removable functional appliances during the retention phase. Am J Orthod Dentofacial Orthop. 2013;144(4):533-540.

30. Hyun P, Preston CB, Al-Jewair TS, Park-Hyun E, Tabbaa S. Patient compliance with Hawley retainers fitted with the SMART ${ }^{\circledR}$ sensor: a prospective clinical pilot study. Angle Orthod. 2015;85(2):263-269. doi:10.2319/030814-163.1.

31. Bos A, Kleverlaan CJ, Hoogstraten J, Prahl-Andersen B, Kuitert R. Comparing subjective and objective measures of headgear compliance. Am J Orthod Dentofacial Orthop. 2007;132(6):801-805.

32. Schott TC, Ludwig B. Microelectronic wear-time documentation of removable orthodontic devices detects heterogeneous wear behavior and individualizes treatment planning. Am J Orthod Dentofacial Orthop. 2014;146(2):155-160.

33. Eppright M, Shroff B, Best AM, Barcoma E, Lindauer SJ. Influence of active reminders on oral hygiene compliance in orthodontic patients. Angle Orthod. 2014;84(2):208-213.

34. Kayser C, Petkov CI, Logothetis NK. Visual modulation of neurons in auditory cortex. Cereb Cortex. 2008;18(7):1560-1574.

clinical outcomes for existing disease states are major areas of interest for the journal. This journal has been accepted for indexing on PubMed Central. The manuscript management system is completely online and includes a very quick and fair peer-review system, which is all easy to use. Visit http://www dovepress.com/testimonials.php to read real quotes from published authors. 